\title{
Eddy current modelling using multi-layer perceptron neural networks for detecting surface cracks
}

\author{
S. Harzallah, R. Rebhi, M.Chabaat \\ University Built Environmental Research Lab., Civil Engineering Faculty, University of Sciences and Technology \\ HouariBoumediene, B.P 32 El Alia Bab Ezzouar, Algiers 16111 Algeria. \\ sharzallab@usthb.dz,r_rebbi@yahoo.fr,m.chabaat@yahoo.com
}

\begin{abstract}
A. Rabehi
Laboratoire de Micro-électronique Appliquée, Université Djillali Liabès de Sidi Bel Abbés, BP 89, 22000 Sidi Bel Abbés, Algeria rab_ebi@hotmail.fr
\end{abstract}

\begin{abstract}
A new method for computing fracture mechanics parameters using computational Eddy Current Modelling by Multi-layer Perceptron Neural Networks for detecting surface cracks. The method is based upon an inverse problem using an Artificial Neural Network (ANN) that simulates mapping between Eddy current signals and crack profiles. Simultaneous use of ANN by MLP can be very helpful for the localization and the shape classification of defects. On the other side, it can be described as the task of reconstructing the cracks and damage in the plate profile of an inspected specimen in order to estimate its material properties. This is accomplished by inverting eddy current probe impedance measurements that are recorded as a function of probe position, excitation frequency or both. In eddy current nondestructive evaluation, this is widely recognized as a complex theoretical problem whose solution is likely to have a significant impact on the detection of cracks in materials
\end{abstract}

KEYWORDS. 3D-FEM-EC; Artificial Neural Network; Inverse Problems; Cracks; Multi-layer Perceptron.

\section{open ACCESS}

Citation:. Harzallah, S., Rebhi, R., Chabaat, M., Rabehi, A., Eddy current modelling using multilayer perceptron neural networks for detecting surface cracks, Frattura ed Integrità Strutturale, 45 (2018) $147-155$.

Received: 07.03 .2018

Accepted: 17.06 .2018

Published: 01.07 .2018

Copyright: $\odot 2018$ This is an open access article under the terms of the CC-BY 4.0, which permits unrestricted use, distribution, and reproduction in any medium, provided the original author and source are credited.

\section{INTRODUCTION}

etection and characterization of a crack in a plate at its initial stage (before propagation), is a real industrial challenge and a major element in security especially in high-risk areas. It is on the basis of these characterizations that engineers have the means then to analyze such crack behavior and predicting its spread, therefore assess its harmfulness as well as the life of the inspected body [1,2]. 
The (ECT) technique works on the principle of electromagnetic induction, and it consists on the detection of the magnetic field due to the eddy current induced on the tested specimen. The presence of the defect modifies the eddy currents pattern and hence gives rise to field perturbation closely related to the position and shape of the defects. The excitation field is carried out by using a coil fed by an alternating current and the changed impedance coil can be computed to account the defect influence on the induced currents. The modeling of a practical configuration of (EC) sensor is generally complex and requires extended analytical or numerical developments. The Finite Element method (FEM) is more general, numerically superior, primarily used for its versatility modeling of material properties, simulations of boundary conditions, modeling arbitrary domain space, and reduces substantially the experimental work [3].

The inverse Eddy current problem can be described as the task of reconstructing the electrical conductivity profile of an inspected specimen in order to estimate its material properties. This is accomplished by inverting Eddy current probe impedance measurements which are recorded as a function of probe position, excitation frequency or both. In eddy current nondestructive evaluation this is widely recognized as a complex theoretical problem whose solution is likely to have a significant impact on the characterization of conductive materials [4].

The Neurons are the fundamental elements in each layer, and every neuron in one layer is associated and interacts with other layers. The outputs of every neuron in the hidden and output layers are determined by the previous output $\left(\Sigma \mathrm{w}_{\mathrm{ij}} \mathrm{x}_{\mathrm{j}}\right.$, $x_{j}$ is the input signals), activation function $\left(f\left(\sum w_{i j} x_{j}\right)\right)$, and weighting coefficients $\left(w_{i j}\right)$ [4]. The MLP network is processed as follows: the information flow is input into the input layer and passes through the hidden and output layers to achieve the output information. The Tan-sigmoid activation function is used in the neurons of hidden and output layers in this work [5].

ANN is composed of highly interconnected processing elements called neurons. These latest can perform arbitrary mappings between sets of input-output pairs. This is achieved by adjustment of the weights of interconnections after training through the presentation of examples. Neural network performance has been proven robustness when faced incomplete, fuzzy or novel data. Previous work has shown that ANN can also be used as for solving electric and/or magnetic inverse problems. ANN is then a tool used to information processing systems which can recognize highly complex patterns within available data. It has recently been applied to solve the electromagnetic non-destructive testing inverse problem and has been proved to have a higher accuracy compared to classical methods [6-8].

\section{FEM DISCRETIZATION OF 3-D EDDY CURRENT GOVERNING EQUATIONS}

7 he eddy current testing phenomenon can be treated as a quasi-static electromagnetic field problem which is expressed by the governing field equations in terms of electrical scalar and magnetic vector potentials. These equations can be solved by 3-D Finite Element Method. Application of the Coulomb gauge allows simultaneous solution of the coupled magnetic vector potential and the electric scalar potential equation in the inductor $\Omega_{\mathrm{i}}$ and the conducting region $\Omega_{c}$, the non-conducting region representing the air $\Omega_{a}$ with a current density source. These equations can be written as $[9,10]$

$$
\nabla \times(v \nabla \times A)-\nabla\left(v_{p} \nabla \cdot A\right)= \begin{cases}0 & \text { in } \Omega_{a} \\ J_{S} & \text { in } \Omega_{i} \\ -J \omega \sigma(A+\nabla . v) & \text { in } \Omega_{a}\end{cases}
$$

$$
\nabla \cdot \sigma(J \omega A+\nabla \cdot v)=0
$$

where, $v_{p}$ the penalty term, $\sigma$ the electric conductivity, $v$ is the magnetic reluctivity and $\mathrm{V}$ is the electrical potential, $\mathrm{J}_{\mathrm{S}}$ the current density source. The integral A - V formulation is obtained when applying Galerkin's methods and the weighted residuals for Eqns. (1) and (2), using vector $\mathrm{Ni}$ and scalar $\alpha_{\mathrm{i}}$ weighted functions. Such a formulation leads to the following integral forms by [11];

$$
\int_{\Omega}\left[v\left(\nabla \times N_{i}\right) \cdot(\nabla \times \mathrm{A})+v_{p}\left(\nabla \cdot N_{i}\right)(\nabla \cdot \mathrm{A})\right] d \Omega=\int_{\Omega}\left[J \omega \sigma\left(N_{i} A+\alpha_{i} \mathrm{~V}\right)\right] d \Omega
$$




$$
\begin{aligned}
& \oint_{\Gamma} N_{i}(\nabla \times \mathrm{A}) d \Gamma=\oint_{\Omega} N_{i} \cdot J_{s} d \Omega \\
& \int_{\Omega} J \omega \sigma\left[\nabla \cdot\left(N_{i} A+\alpha_{i} \nabla \mathrm{V}\right)\right] d \Omega=0
\end{aligned}
$$

\section{SENSOR IMPEDANCE CALCULATION}

he detection of change of the resulting magnetic field is based on two basic methods: the NDT differential mode represented by two separate coils linked magnetically and supplied by the same current and the NDE absolute mode which makes use of only one coil. The impedance variation is obtained from comparison with the reference impedance. The impedance variation $Z$ is a complex number. The imaginary part is computed with the magnetic energy (WM) in the whole meshed domain and the real part is computed with the Joule Losses in the conductive media and the imaginary part is computed with the magnetic energy in the whole meshed domain [12].

The coil impedance with an excited current $\mathrm{I}$ at a frequency $\mathrm{F}$ is obtained by the following expression;

$$
\begin{aligned}
& \left\{\begin{array} { l } 
{ \operatorname { R e } ( Z ) = \frac { J L } { I ^ { 2 } } } \\
{ \operatorname { R e } ( \Delta Z ) = \int _ { \Omega _ { C } } \frac { 1 } { \sigma } ( | J _ { f } ^ { 2 } | - | J | ^ { 2 } ) d \Omega }
\end{array} \text { and } \left\{\begin{array}{l}
\operatorname{Im}(Z)=\frac{\omega W_{M}}{I^{2}} \\
\operatorname{Im}(\Delta Z)=\omega \int_{\Omega_{c}} \frac{1}{\mu}\left(\left|B_{f}^{2}\right|-|B|^{2}\right)
\end{array}\right.\right. \\
& \left\{\begin{array}{l}
Z=R+j X \\
Z=\frac{1}{I^{2}}\left(\int_{\Omega} \frac{J^{2}}{\sigma} d \Omega+j 2 \pi F \int_{\Omega}(B . H) d \Omega\right.
\end{array}\right.
\end{aligned}
$$

where $\mathrm{J}, \mathrm{B}$, and $\mathrm{H}$ are the induced magnetic induction, and the magnetic field, respectively [13].

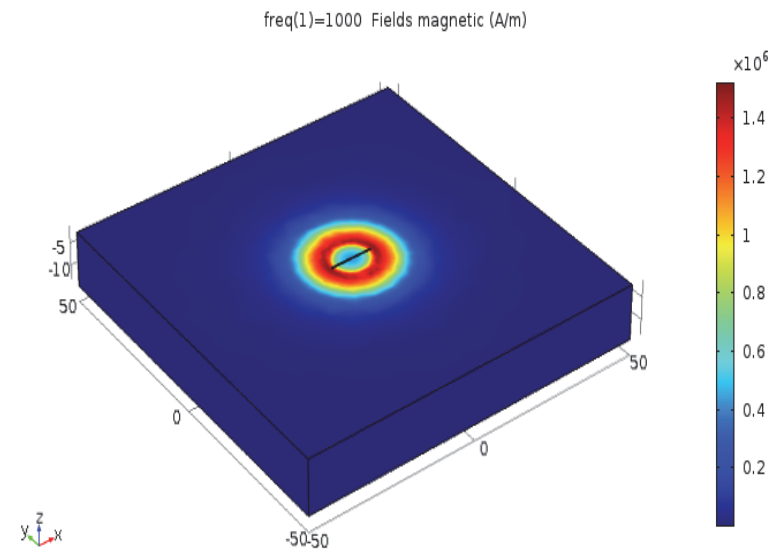

Figure 1: Vector of induction B.

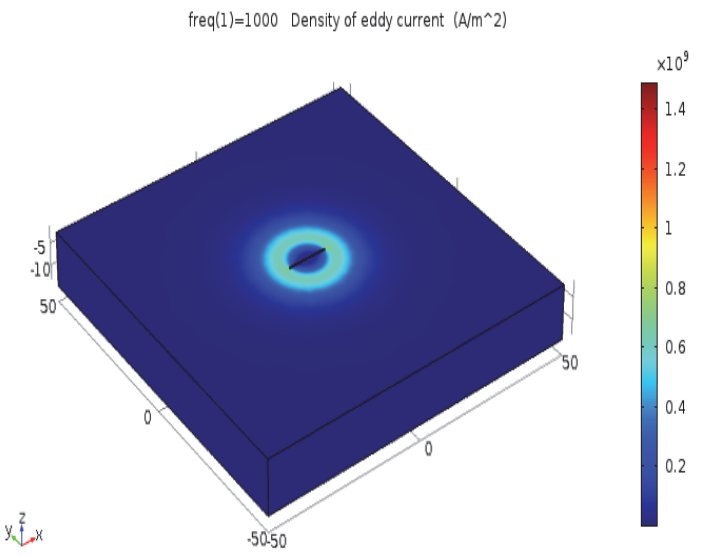

Figure 2: Density of the induced current.

\section{Applications}

or the application, we chose to test a magnetic plate without any crack and characterized by a high conductivity $10^{7}$ Sm-1 with a magnetic permeability $\mu$ of $1.210^{-6} \mathrm{H} / \mathrm{m}$. The plate is excited by a sinusoidal current of density $\mathrm{J}=2.67$ $10^{6} \mathrm{~A} / \mathrm{m}$ and a frequency of $10 \mathrm{Khz}$. EC testing problem deals with a Pancake coil placed above a flat plate, as 
shown in Fig. 2, with rectangular crack. The coil is placed along the crack length direction, moving in the XOY Plan, parallel to the $\mathrm{x}$-axis[9].

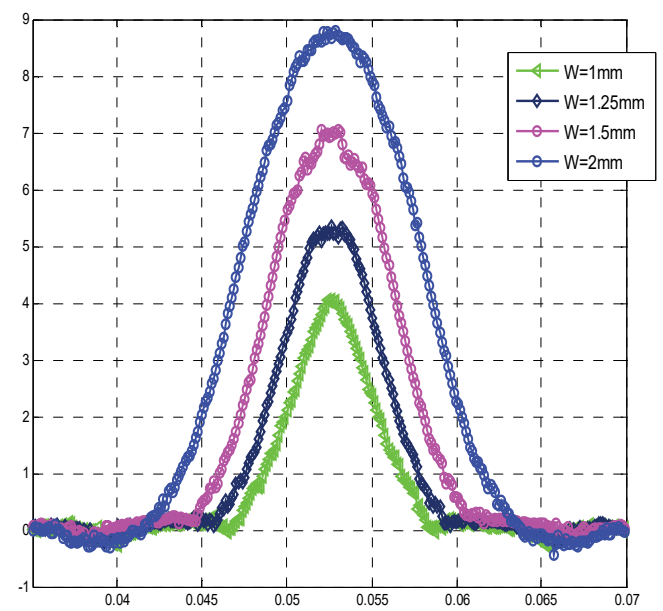

Figure 3: Impedance Z vs. the crack's width.

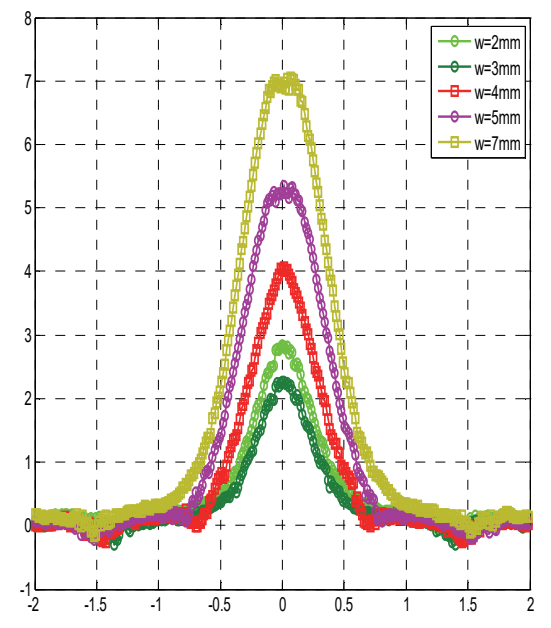

Figure 4: Impedance Z vs. the crack's depth.

\section{RESULTS INTERPRETATION}

he results of simulation obtained in the case of a non-magnetic plate without defects are illustrated in the following Figures;

- Fig. 1 represents the distribution of the currents induced on the surface of target. It is noticed that their values are high because the conductivity of the non-magnetic target is large $(j=\sigma .2 \pi$.f.A) but are relatively weak compared to the primary currents.

- Fig. 2 indicates the distribution of magnetic field. That explains the strong concentration of the vectors of magnetic induction on the level of the cracks without the possibility to penetrate inside the plate due to the characteristics of material and the effect of the frequency.

- In Figs. 3 and 4, one can notice that when the width decreases then, the value of impedance $\Delta Z$ decreases at the same rate. The width of the defect has a great influence on the variation of impedance meanwhile the variation depth of the defect has a light influence on $\Delta \mathrm{Z}$. It is noticed that the difference of impedance $\Delta \mathrm{Z}$ has a dependency on the width of the defect, indeed the width of defect increases $\Delta Z$. On the other side, when the width decreases then, $\Delta Z$ decreases. The depth of defect has an effect on the impedance. The variation depth of the defect has a light influence on $\Delta Z$. It is noticed that the difference of impedance $\Delta Z$ dependents on the width of the defect. The width of defect increases $\Delta Z$ and also one can remark that when the width decreases then, $\Delta Z$ decreases at the same rate. It is obvious that the depth of defect does not influence the impedance.

\section{Artificial Neural Networks}

A lthough this is an oversimplified model of the biological brain, the organization and the information processing strategies of an ANN are based on the features of their biological counterparts. The neurons combine the input impulses in several ways, operating in parallel with other neurons to perform a variety of functions. In artificial neural nets, each simple node performs a weighted sum of the inputs and computes a nonlinear function of the results [17]. The prediction methodology by artificial intelligence or machine learning is based on the following key steps [1]:

1. Selection of the variable to predict, e.g. global, diffuse or beam solar radiation on horizontal or tilted surface;

2. Selection of the input parameters and data collection, e.g. climatologic data, geographical coordinates, past time series, etc.;

3. Definition of the training and testing sets;

4. Development of the ANN model and training phase assessment; 
5. Calculation of the prediction error, through statistical indices, adopting the testing set;

6. Comparisons and final choice of the best ANN configuration.

\section{MATERIAL AND METHOD}

$\mathrm{N}$ eural networks can be classified into dynamic (e.g. Elman) and static (e.g. MLP) categories. MLP is the most commonly used static networks [18], in which the input is presented to the network along with the desired output, and the weights are adjusted so that the network attempts to produce the desired output. The MLP network has three layers of neurons (nodes) an input layer, a hidden layer and an output layer (Fig. 5).

The Neurons are the fundamental elements in each layer, and every neuron in one layer is associated and interacts with other layers. The outputs of every neuron in the hidden and output layers are determined by the previous output $\left(\Sigma \mathrm{w}_{\mathrm{ij}} \mathrm{x}_{\mathrm{j}}\right.$ , $x_{j}$ is the input signals), activation function $\left(f\left(\Sigma w_{i j} x_{j}\right)\right)$, and weighting coefficients $\left(w_{i j}\right)$ [19]. The MLP network is processed as follows: the information flow is input into the input layer and passes through the hidden and output layers to achieve the output information. The Tan-sigmoid activation function is used in the neurons of hidden and output layers in this work. In detail, neurons sum the weight-controlled input production to apply the nonlinear activation function as follows:

$$
\begin{aligned}
& \mathrm{a}_{\mathrm{i}}=\sum_{\mathrm{j}=1}^{\mathrm{n}} \mathrm{w}_{\mathrm{ij}} \mathrm{x}_{\mathrm{j}} \\
& \mathrm{y}_{\mathrm{i}}=\mathrm{f}\left(\mathrm{a}_{\mathrm{i}}\right)=\frac{2}{1+\mathrm{e}^{-2 \mathrm{a}_{\mathrm{i}}}}-1
\end{aligned}
$$

where $\mathrm{w}_{\mathrm{ij}}$ is the weight coefficient, $\mathrm{x}_{\mathrm{j}}$ is the input signals, $\mathrm{a}_{\mathrm{i}}$ is the summation of the weighted inputs, and $\mathrm{y}_{\mathrm{i}}$. is the output at neuron $i$ of the output layer.

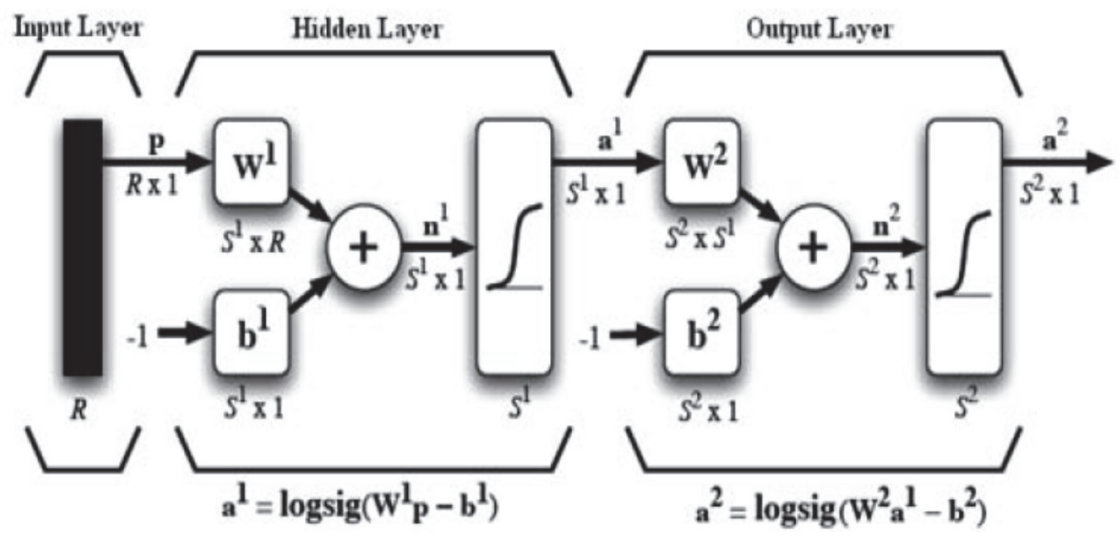

Figure 5: Architecture of MLP neural network.

\section{MODEL VALIDATION}

$\mathrm{F}$ or the estimation of the depth and length, a type of neural network (MLP) is used. Fig. 6 represents the steps of inversion.

The accuracy and performance of the derived correlations in predicting of global solar radiation was evaluated on the basis of the following statistical error tests which are coefficient of determination $\mathrm{R}^{2}$, root mean square error (RMSE) and its normalised value (nMBE), relative root mean square error (rRMSE), mean absolute error (MAE) and its normalised value (nMAE). These error indices are defined as [20,21]: 


$$
\begin{aligned}
& \text { RMSE }=\sqrt{\frac{1}{n} \sum_{i=1}^{n}\left(y_{i}-x_{i}\right)^{2}} \\
& \text { rRMSE }=\frac{\sqrt{\frac{1}{n} \sum_{i=1}^{n}\left(y_{i}-x_{i}\right)^{2}}}{\frac{1}{N} \sum_{i=1}^{n} x_{i}} \times 100
\end{aligned}
$$

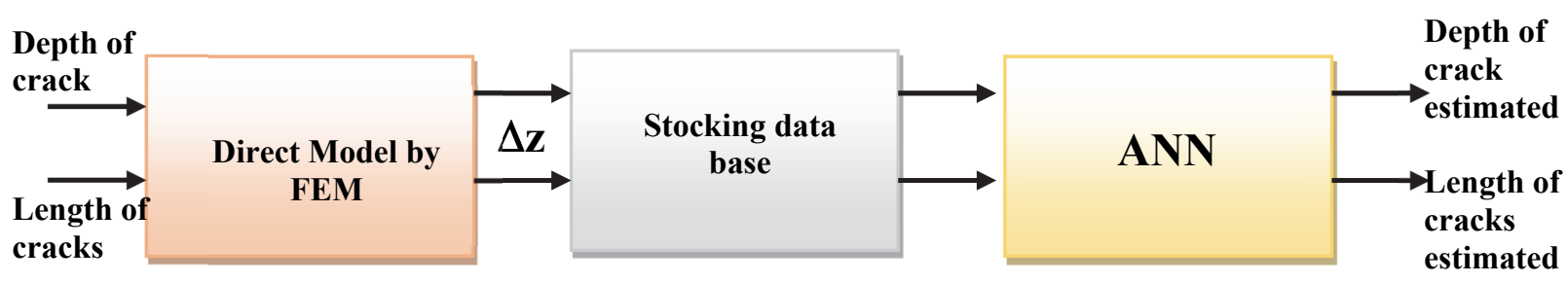

\section{X position}

Figure 6: Inverse problem RN with two inputs and two outputs.

The ranges of rRMSE define the model performance as:

$$
\begin{aligned}
& \text { Excellent if: } \quad \text { rRMSE }<10 \% \\
& \text { Good if: } \quad 10 \%<\text { rRMSE }<20 \% \\
& \text { Poor if: } \quad \text { rRMSE }>30 \% \\
& M A E=\frac{1}{\mathrm{~N}} \sum_{\mathrm{i}=1}^{\mathrm{n}}\left|\mathrm{y}_{\mathrm{i}}-\mathrm{x}_{\mathrm{i}}\right| \\
& R^{2}=\frac{\sum_{i=1}^{n}\left(y_{i}-x_{i}\right)^{2}}{\sum_{i=1}^{n}\left(y_{i}-\bar{y}_{i}\right)^{2}}
\end{aligned}
$$$$
\text { Fair if: } \quad 12 \%<\text { rRMSE }<30 \%
$$

where: $\mathrm{y}_{\mathrm{i}}$ and $\mathrm{x}_{\mathrm{i}}$ are the estimated and the measured values, $\overline{\mathrm{y}}_{\mathrm{i}}$ is the average of estimated values and $\mathrm{N}$ is data number.

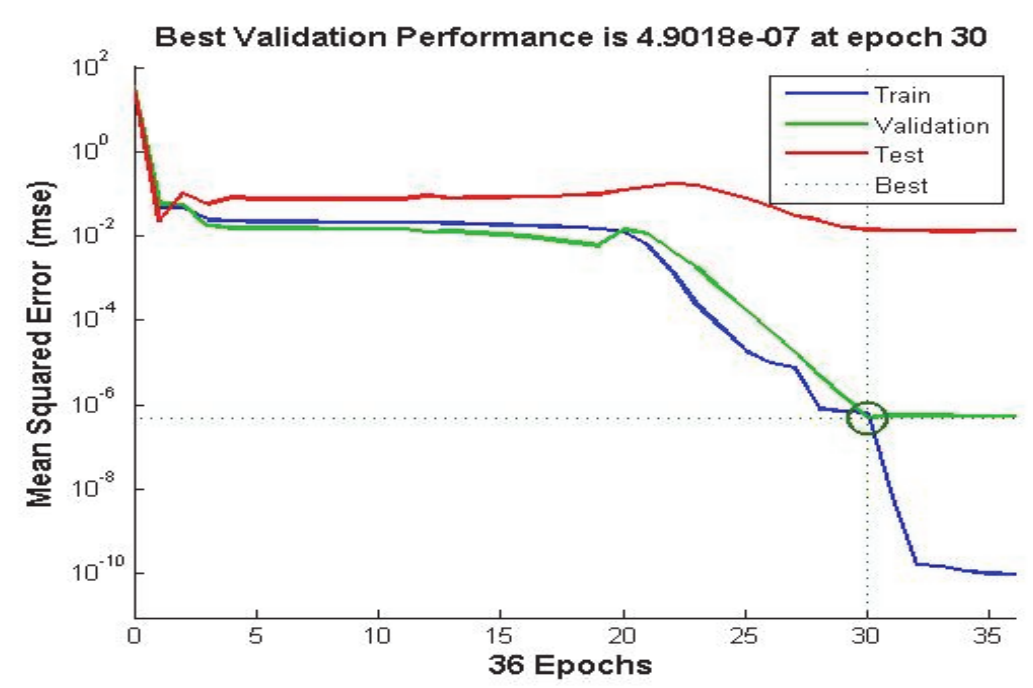

Figure 7: Cost function (MSE) vs. number of iterations. 
Evaluation of the depth or length independently does not lead to a complete characterization of the geometry of such a crack. This is why an evaluation of two sizes (depth and length) will be established at the same time exploiting the previous architecture; but this time the database must contain two vectors of inputs and two vectors for output to estimate. Fig. (6) shows a block diagram of a network (MLP) with two inputs and two outputs for the fault characterization. After repeated several times the learning algorithm (for each iteration biases reset, obtained results are different), Fig. (7) is drawn showing the cost function (MSE). One can notice that it converges to the imposed optimum of $5.10^{-7}$ after 122 iterations.

Curves in Figs. 8 and 9 give an acceptable correlation between the rectangular geometry of a crack by the direct model using the finite element method and those obtained by the neural network and mean absolute error (MAE).
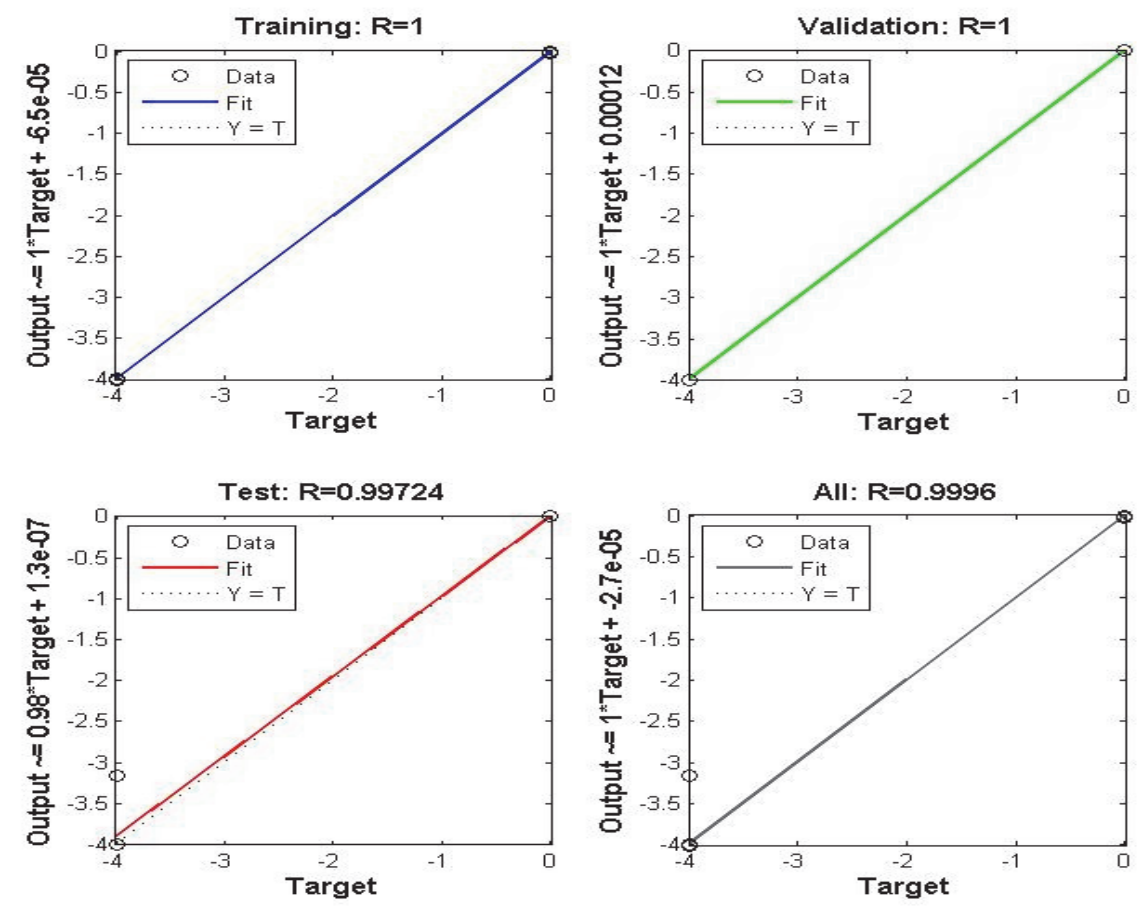

Figure 8: The correlation between the desired exit and the exit of the network.

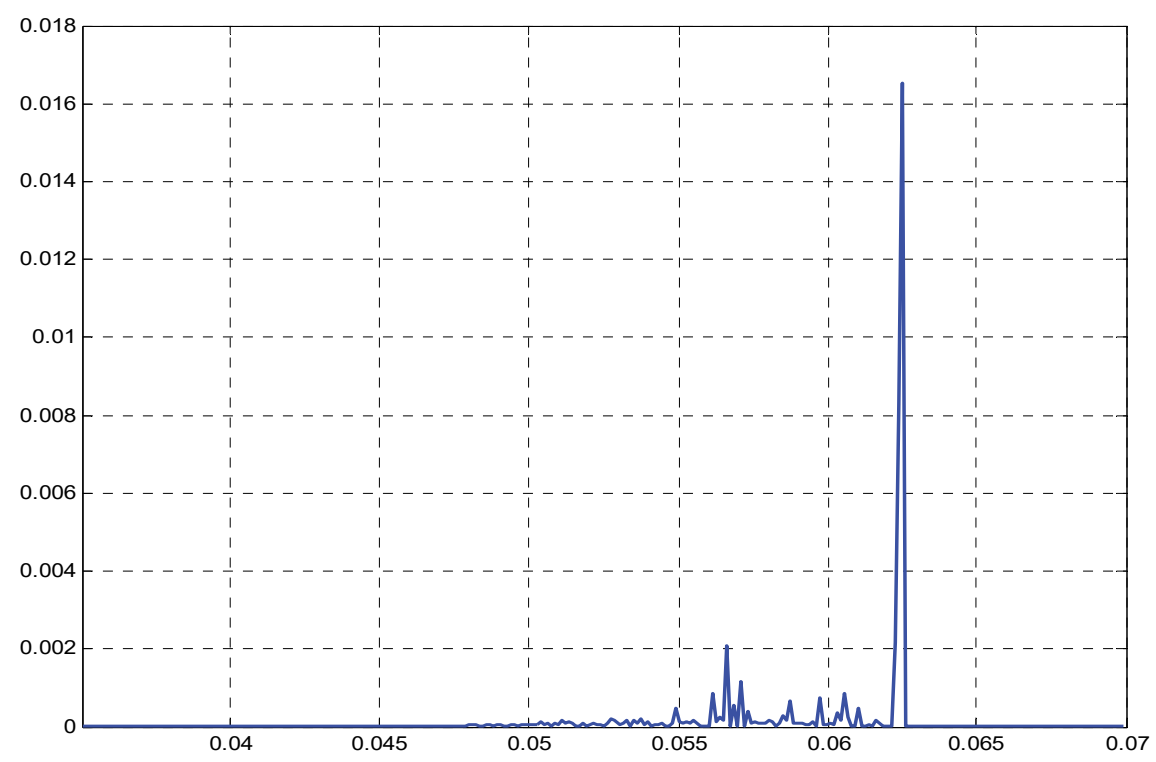

Figure 9: Mean absolute error (MAE). 
Results from the FEM for every figure have been inversed just for comparisons purposes with the ANN optimization and the true profile of the shape crack. These comparisons are shown in the Fig. 10 and give good maps representing the NN in very short time. However, the approach described in the paper identifies the problem fairly and effectively of admissible solutions for different shapes.
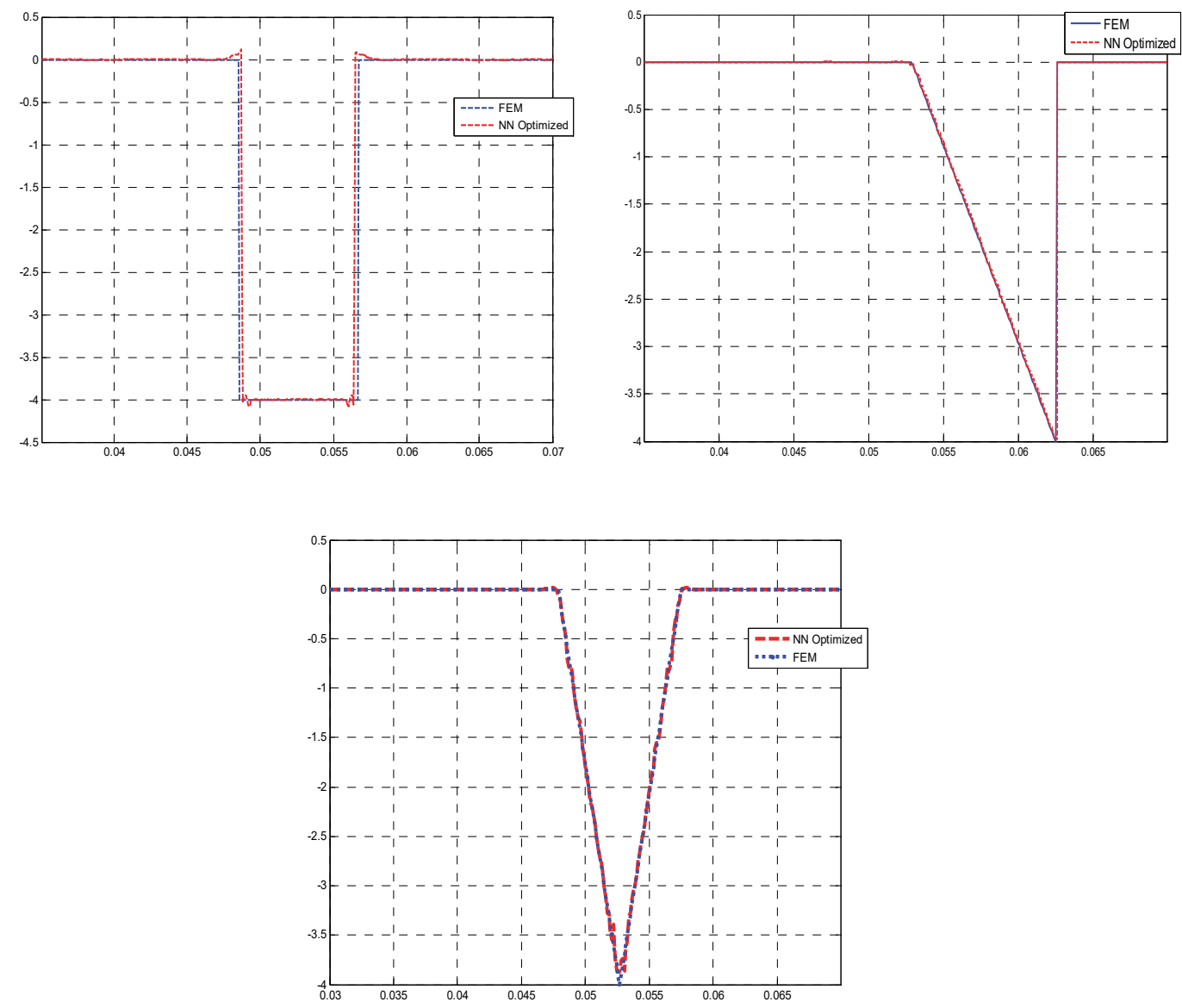

Figure 10: Comparison results from the NN Optimized, FEM profile.

\section{CONCLUSION}

A neural network approach for solving inverse problem in eddy current testing is presented in this paper. The main idea is the introduction of a categorization for the shape reconstruction using a neural network. Results are obtained for a simple eddy current problem using FEM method as an experimental support. On the other hand, NN responses which estimate the forms of cracks are given by the inverse model. It is shown herein that the application of artificial intelligence can be a good substitute or a help of the NDT operators' work.

\section{REFERENCES}

[1] Harzallah, S. Chabaat, M. and Chabane, K. (2017). Numerical study of eddy current by Finite Element Method for cracks detection in structures, Frattura ed Integrità Strutturale, 39, pp. 282-290. DOI: 10.3221/IGF-ESIS.39.26.

[2] Hamia, R., Cordier, C. and Dolabdjian, C. (2014). Eddy-current non-destructive testing system for the determination of crack orientation, NDT \& E International, 61, pp.. 24-28.

[3] Harzallah, S. and Chabaat, M. (2017). 3D-FEM computation and experimental study of eddy currents for characterization of surface cracks. Int. J. of Structural Integrity, 8(5), pp.603-610, DOI: 10.1108/IJSI-02-2017-0013. 
[4] Zaoui, A., Menana, H., Feliachi, M. and Berthiau, G. (2010). Inverse problem in nondestructive testing using arrayed eddy current sensors, Journal of Sensors, 10(9), pp. 8696-8704.

[5] Helifa, B., Filiachi, M., Lefkaier, I.K., Boubenider, F., Zaoui, A. and Lagraa, N. (2016). Characterization of surface cracks using eddy current NDT simulation by 3D-FEM and inversion by neural network, ACES Journal, 31(2).

[6] Rao, B.P.C., Raj, B. and Kröning, M. (1999). Artificial Neural Network for On-Line Eddy Current Testing. In: Thompson D.O., Chimenti D.E. (eds) Review of Progress in Quantitative Nondestructive Evaluation. Review of Progress in Quantitative Nondestructive Evaluation, Springer, Boston, MA, 18 A.

[7] Harzallah, S., Mimouni, M. L., Benissad, S. and Chabaat, M. (2018). 3D-FEM Computational and Inverse Problem in Nondestructive Evaluation Using Neural Networks for Detection of Cracks, Transylvanian Review, 26, pp. 1-13.

[8] Uchimoto, T., Takagi, T., Ichihara, T. and Dobmann, G. (2015). Evaluation of fatigue cracks by an angle beam EMATET dual probe, NDT \& E International, 72, pp. 10-16.

[9] Harzallah, S. and Chabaat, M. (2016). 3-D Eddy current modeling for evaluating the Fracture parameters by a new method based on the variation of the impedance, International Journal of Applied Electromagnetics and Mechanics, 53 , pp. $1-15$.

[10] Sakagami, T. (2015). Remote nondestructive evaluation technique using infrared thermography for fatigue cracks in steel bridges, Fracture of Engineering Materials \& Structures, 38(7).

[11] Notghi, B. and Brigham, J. C. A. (2015). Computational approach for robust nondestructive test design maximizing characterization capabilities for solids and structures subject to uncertainty, International Journal for Numerical Methods in Engineering 104(4).

[12] Zaidi, H., Santandre, L., Krebsa, G. and Le Bihan, Y. (2014). Finite element simulation of the probe displacement in eddy current testing, International Journal of Applied Electromagnetics and Mechanics, 45, pp. 887-893.

[13] Garcia-Martin, J., Gomez-Gil, J. and Vazquez-Sanchez, E. (2011). Non-Destructive Techniques based on eddy current testing, Sensors, 11(3), pp. 2525-2565.

[14] Hughes, R., Fan, Y. and Dixon, S. (2014). Near electrical resonance signal enhancement (NERSE) in eddy current crack detection, Journal of Nondestructive Testing and Evaluation, 66, pp. 82-89.

[15] Babbar, V.K., Underhill, P.R., Stott, C. and Krause, T.W. (2014). Finite element modelling of second layer crack detection in aircraft bolt holes with ferrous fasteners present, 65, pp. 64-71.

[16] Xu, P., Huang, S. and Zhao, W. (2011). A new differential eddy current testing sensor used for detecting crack extension direction, NDT\&E International, 44, pp. 339-343.

[17] Bortolini, M., Gamberi, M. and Regattieri, A. (2016). Artificial neural network optimisation for monthly average daily global solar radiation prediction. Energy Conversion and Management, 120, pp. 320-329.

[18] Moghaddamnia, A., Remesan, R., Kashani, M. H., Mohammadi, M., Han, D. and Piri, J. (2009). Comparison of LLR, MLP, Elman, NNARX and ANFIS Models_-with a case study in solar radiation estimation. Journal of Atmospheric and Solar-Terrestrial Physics, 71(8), pp. 975-982.

[19] Hassanpour Kashani, M. (2008). Flood estimation at ungauged sites using a new hybrid model. Journal of Applied Sciences 9, pp. 1744-1749.

[20] Guermoui, M., Rabehi, A., Benkaciali, S. and Djafer, D. (2016). Daily global solar radiation modelling using multi-layer perceptron neural networks in semi-arid region. Leonardo Electronic Journal of Practices and Technologies, 28, pp. 35-46.

[21] Rabehi, A., Guermoui, M., Djafer, D. and Zaiani, M. (2015). Radial basis function neural networks model to estimate global solar radiation in semi-arid area. Leonardo Electronic Journal of Practices and Technologies, 27, pp. 177-184. 